\title{
Enhanced Transmit-Antenna Selection Schemes for Multiuser Massive MIMO Systems
}

\author{
Byung-Jin Lee, ${ }^{1}$ Sang-Lim Ju, ${ }^{1}$ Nam-il Kim, ${ }^{2}$ and Kyung-Seok Kim ${ }^{1}$ \\ ${ }^{1}$ Smart Radio Communication System Lab., Department of Electrical and Electronic Engineering, Chungbuk National University, \\ Cheongju, Chungbuk 28644, Republic of Korea \\ ${ }^{2} 5$ G Giga Communication Research Laboratory, Electronics and Telecommunications Research Institute, \\ Daejeon 34129, Republic of Korea \\ Correspondence should be addressed to Kyung-Seok Kim; kseokkim@cbnu.ac.kr
}

Received 7 February 2017; Revised 5 May 2017; Accepted 22 May 2017; Published 18 June 2017

Academic Editor: Jung H. Lee

Copyright (C) 2017 Byung-Jin Lee et al. This is an open access article distributed under the Creative Commons Attribution License, which permits unrestricted use, distribution, and reproduction in any medium, provided the original work is properly cited.

Massive multiple-input multiple-output (MIMO) systems are a core technology designed to achieve the performance objectives defined for $5 \mathrm{G}$ wireless communications. They achieve high spectral efficiency, reliability, and diversity gain. However, the many radio frequency chains required in base stations equipped with a high number of transmit antennas imply high hardware costs and computational complexity. Therefore, in this paper, we investigate the use of a transmit-antenna selection scheme, with which the number of required radio frequency chains in the base station can be reduced. This paper proposes two efficient transmit-antenna selection (TAS) schemes designed to consider a trade-off between performance and computational complexity in massive MIMO systems. The spectral efficiency and computational complexity of the proposed schemes are analyzed and compared with existing TAS schemes, showing that the proposed algorithms increase the TAS performance and can be used in practical systems. Additionally, the obtained results enable a better understanding of how TAS affects massive MIMO systems.

\section{Introduction}

The demand for data traffic has soared dramatically as a result of the explosion of the demand for Internet services, high-definition video calls, and live streaming, fueled by the worldwide levels of smartphone deployment [1]. Future developments will only increase the data traffic requirements. The massive multiple-input multiple-output (MIMO) technique, which uses a large number of antennas to receive and transmit a large number of concurrent streams, is one of the main technologies addressing this challenge $[2,3]$.

Massive MIMO enables highly efficient transmissions by using at least 64 antennas per base station (BS) $[4,5]$. However, to accommodate these numerous antennas, the same number of radio frequency (RF) chains is needed at the BS. Therefore, as the number of antennas increases, the hardware complexity and processing costs of the BS increase exponentially. Additionally, as the number of antennas increases, the amount of computation for calculating the
BS precoding vector values also increases. To alleviate these problems, an antenna selection method has been studied [6], which can implement fewer RF stages than the number of available transmit antennas by selecting the specific transmit antennas to be used with the (reduced number of) RF stages. The typical transmission antenna selection scheme proceeds by exhaustive search, selecting the best possible subset (combination) of the full set of antennas [7]. Even though the performance of this exhaustive search can be optimized, its complexity still increases linearly with the total number of antennas and the number of antennas to be selected. Therefore, much research on transmission antenna selection schemes has been performed, in an attempt to achieve lower complexity than that of exhaustive search methods [8-12]. With that purpose, greedy search schemes were proposed in [11]. The simplest method is to select the transmitting antenna by computing the norm of the thermal vector of the entire channel; however, instead of simply reducing complexity, this also reduces the user's achievable rate of transmission. 


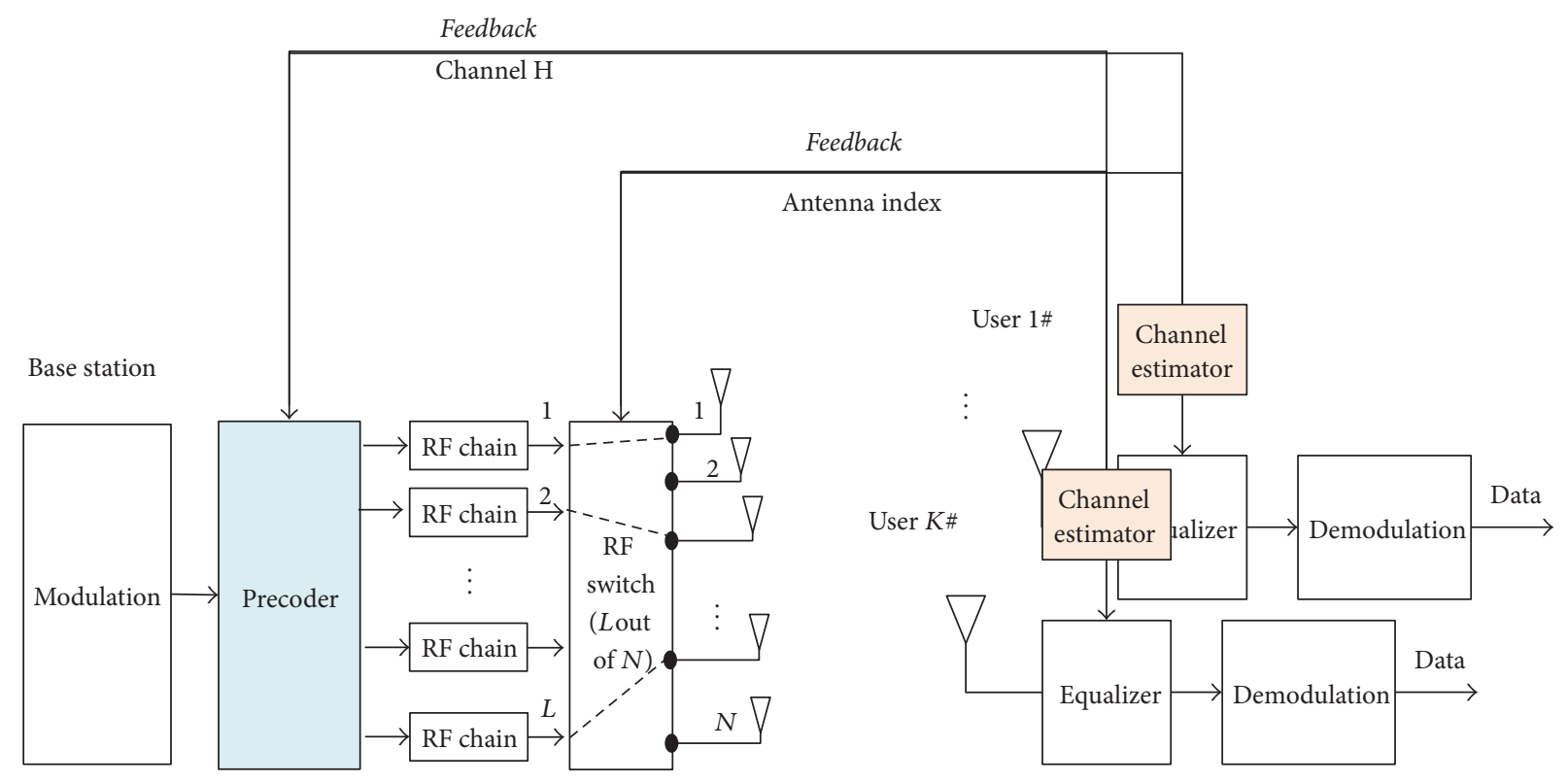

FIGURE 1: Multiuser massive MIMO system model.

In this paper, we propose a transmit-antenna selection scheme that considers both complexity and performance. Focusing on the need to address the explosive demand for data traffic, we suggest ways to maximize performance, even if at the cost of slightly increasing complexity. Two schemes are proposed to generate candidate groups of antennas and then select the best candidate: a capacity-based scheme and a norm-based scheme. This paper is organized as follows. Section 2 discusses the massive MIMO system model. Section 3 presents the proposed antenna selection schemes. Section 4 analyzes the spectral efficiency of the proposed schemes and compares it with that of existing schemes. Section 5 concludes the paper.

\section{System Model}

In this paper, we consider downlink massive MIMO systems with one BS ( $N$ transmit antennas) and $K$ users (one receive antenna per user), as shown in Figure 1. For cost and complexity reasons the BS has only $L$ RF chains $(N \geq L \geq K)$; the data must be transmitted by selecting only $L$ of the total $N$ antennas. In this paper, we consider that the number of RF chains in the BS and the number of users are the same $(L=K)$. In the case of large antenna systems, zero-forcing (ZF), which is a simple linear precoding technique capable of achieving an almost optimal performance [3], was applied. In multiuser massive MIMO systems, the signals received by users can be expressed as follows:

$$
Y=H x+n_{0}
$$

where $H \in C^{K \times N}$ is the channel matrix, given by

$$
\begin{aligned}
H & =\left[\begin{array}{ccccc}
h_{11} & \cdots & h_{1 n} & \cdots & h_{1 N} \\
\vdots & & & & \vdots \\
h_{k 1} & & \ddots & & h_{k N} \\
\vdots & & & \vdots \\
h_{K 1} & \cdots & h_{K n} & \cdots & h_{K N}
\end{array}\right] \\
& =\left[H_{\bar{K} 1}, H_{\bar{K} 2}, \ldots, H_{\bar{K} N}\right] .
\end{aligned}
$$

In (2), $h_{k n}$ is a channel element from the $n$th transmitantenna to the $k$ th user $(n=1,2, \ldots, N ; k=1,2, \ldots, K) ; H_{\bar{K} n}$ denotes the $K \times 1$ channel vector between the $n$th transmitantenna and the $K$ users. It is assumed that the elements of $H$ can be modeled as independent and identically distributed (iid) complex Gaussian random variables with zero-mean and unit-variance (Rayleigh fading). Moreover, the users are considered to have full knowledge of the channel matrix $H$ and transmit it to the BS without any errors or time delays. The term $n_{0}$ is additive white Gaussian noise (AWGN), and $x$ is the $\mathrm{ZF}$ coding signal vector, which is expressed as follows [13]:

$$
x=\frac{1}{\sqrt{\gamma}} V q
$$

where $q=\left[q_{1}, q_{2}, \ldots, q_{K}\right]^{T}$ is the ZF precoding matrix, $V=$ $H\left(H^{H} H\right)^{-1}, V \in C^{N \times K}$, and $\gamma$ is $\operatorname{Tr}\left\{\left(H H^{H}\right)^{-1}\right\}$, with $(\cdot)^{H}$ denoting the Hermitian transpose operator. To ensure that 
$E\left\{\|x\|^{2}\right\}=1$ when the total BS transmission power is $P_{d}$, a power normalization factor $\Gamma$ is defined by

$$
\Gamma=\frac{P_{d}}{\gamma}
$$

Considering (1)-(4), the signals received by the $k$ th user can also be expressed as

$$
\begin{aligned}
& y_{k}=H_{k \bar{N}} V q+n_{k}, \quad k=1,2, \ldots, K \\
& y_{k}=H_{k \bar{N}} v_{k} q_{k}+H_{k \bar{N}} \sum_{i=1, i \neq k}^{K} v_{i} q_{i}+n_{k} .
\end{aligned}
$$

Assuming that the received signal to noise ratio (SNR) is the same for all users, the received SNR is given by

$$
\mathrm{SNR}=\frac{\Gamma}{\sigma_{n}^{2}}=\frac{P_{d}}{\operatorname{Tr}\left\{\left(H_{k \bar{N}} H_{k \bar{N}}^{H}\right)^{-1}\right\} \sigma_{n}^{2}} .
$$

\section{Proposed Antenna Selection Schemes}

Massive MIMO systems have many channels-because of the large number of antennas-possibly differing in strength. This implies that some antennas can be more useful than others. In this case, different antennas (with different characteristics) can be selected, considering the established design goals. We propose two transmit-antenna selection schemes to enhance the set of existing antenna selection schemes. Both proposed schemes proceed in two steps: first, candidate transmit-antenna subsets are generated; then, an optimal transmit-antenna subset is selected from among the candidates.

Step 1 (generate the transmit-antenna subsets). In this first step, candidate transmit-antenna subsets are generatedusing either a capacity-based or a norm-based approachconsidering both computational complexity and performance. The proposed methods are named capacity-based reduced-complexity exhaustive search (CRCES) and normbased reduced-complexity exhaustive search (NRCES).

(1) CRCES Method. To generate the candidate transmitantenna subsets, instead of computing the capacity for all possible combinations of $L$ selected antennas (as would be done in the optimal selection procedure), the proposed algorithm starts with an empty set of selected antennas and then adds one antenna per step. The method for constructing the subset by considering capacity is as follows. First, we determine the index of the column vector $H_{\bar{K} i}$ with the largest channel capacity $C\left(H_{\bar{K} i}, \rho\right), i=1,2, \ldots, N$, using

$$
\begin{aligned}
C\left(H_{\bar{K} i}, \rho\right) & =\log _{2} \operatorname{det}\left(I_{K}+\rho H_{\bar{K} i} H_{\bar{K} i}^{H}\right), \quad i \in N, \\
P_{1} & =\underset{i \in N}{\arg \max }\left\{C\left(H_{\bar{K} i}, \rho\right)\right\},
\end{aligned}
$$

where $\rho$ is the received SNR. The obtained value of $P_{1}$ is the index of the first optimal transmission antenna. Then, the channel capacity of $H_{\bar{K}\left(P_{1}, i\right)}$ matrix $\left(i \neq P_{1}, i \in N\right)$ including $H_{\bar{K} P_{1}}$ vector is calculated, to select the second antenna index $P_{2}$ having maximum channel capacity. The channel vector for the transmitting antenna is selected in the same way. The $L$ th antenna is selected using

$$
\begin{aligned}
C & \left(H_{\bar{K}\left(P_{1}, P_{2}, \ldots, P_{L-1}, i\right)}, \rho\right) \\
& =\log _{2} \operatorname{det}\left(I_{K}+\frac{\rho}{L} H_{\bar{K}\left(P_{1}, P_{2}, \ldots, P_{L-1}, i\right)} H_{\bar{K}\left(P_{1}, P_{2}, \ldots, P_{L-1}, i\right)}^{H}\right), \\
P_{L} & =\underset{i \neq P_{1}, P_{2}, \ldots, P_{L-1}}{\arg \max }\left\{C\left(H_{\bar{K}\left(P_{1}, \ldots, P_{L-1}, i\right)}, \rho\right)\right\} .
\end{aligned}
$$

Following the above procedure, the antenna indexes from $P_{1}$ to $P_{N}$ are sequentially determined. The entire matrix $H$ is then rearranged according to the index, as follows:

$$
H_{\bar{K}\left(P_{1}, P_{2}, \ldots, P_{N}\right)}=\left[\begin{array}{llll}
\widetilde{H}_{\bar{K} 1} & \widetilde{H}_{\bar{K} 2} & \cdots & \widetilde{H}_{\bar{K} N}
\end{array}\right]=\widetilde{H}_{\text {CRCES }} .
$$

Next, given that the required number of selected transmit antennas is $L$, the transmit-antenna subsets composed of $L$ transmit antennas are investigated. $S_{\text {CRCES }}^{1}$ is the first transmit-antenna subset and is composed of $\widetilde{H}_{\bar{K} 1}$ to $\widetilde{H}_{\bar{K} L}$ columns of $\widetilde{H}_{\text {CRCES }}$; that is,

$$
S_{\text {CRCES }}^{1}=\left[\widetilde{H}_{\bar{K} 1}, \widetilde{H}_{\bar{K} 2}, \ldots, \widetilde{H}_{\bar{K} L}\right] .
$$

The second transmit-antenna subset, $S_{\mathrm{CRCES}}^{2}$, is composed of $\widetilde{H}_{\bar{K} 2}$ to $\widetilde{H}_{\bar{K}(L+1)}$. In the same way, a total of $\zeta$ transmitantenna subsets are obtained, with $\zeta=N-L+1$. In particular, the $j$ th transmit-antenna subset is composed of the following:

$$
S_{\text {CRCES }}^{j}=\left[\widetilde{H}_{\bar{K} j}, \widetilde{H}_{\bar{K}(j+1)}, \ldots, \widetilde{H}_{\bar{K}(j+L-1)}\right], \quad j \in \zeta .
$$

(2) NRCES Method. In this method, the norm of each column of the channel matrix is first calculated as follows:

$$
\left\|H_{\bar{K} n}\right\|^{2}=\sum_{i=1}^{K}\left|h_{i n}\right|^{2}=\left|h_{1 n}\right|^{2}+\left|h_{2 n}\right|^{2}+\cdots+\left|h_{K n}\right|^{2} .
$$

The columns of $H$ are then sorted in descending order, resulting in the new matrix

$$
\widetilde{H}_{\text {NRCES }}=\left[\begin{array}{llll}
\widetilde{H}_{\bar{K} 1} & \widetilde{H}_{\bar{K} 2} & \cdots & \widetilde{H}_{\bar{K} N}
\end{array}\right],
$$

where

$$
\begin{aligned}
\left\|\widetilde{H}_{\bar{K} 1}\right\|^{2} \gg\left\|\widetilde{H}_{\bar{K} 2}\right\|^{2} \gg \cdots \gg\left\|\widetilde{H}_{\bar{K} L}\right\|^{2} \gg \cdots \\
\gg\left\|\widetilde{H}_{\bar{K} N}\right\|^{2} .
\end{aligned}
$$

The transmit-antenna subsets are obtained from $\widetilde{H}_{\text {NRCES }}$ in the same way used with $\widetilde{H}_{\mathrm{CRCES}}$ : the first transmit-antenna subset $S_{\text {NRCES }}^{1}$ (of size $K \times L$ ) is constituted by columns $\widetilde{H}_{\bar{K} 1}$ to $\widetilde{H}_{\bar{K} L}$ of $\widetilde{H}_{\text {NRCES }}$, and the $j$ th transmit-antenna subset, $S_{\text {NRCES }}^{j}$, is given by

$$
S_{\text {NRCES }}^{j}=\left[\widetilde{H}_{\bar{K} j}, \widetilde{H}_{\bar{K}(j+1)}, \ldots, \widetilde{H}_{\bar{K}(j+L-1)}\right] .
$$


Step 2 (select the optimal transmit-antenna subset among the candidate transmit-antenna subsets). This paper selects the optimal transmit-antenna subset (the one that maximizes the user received SNR) among the transmit-antenna subsets generated in the first step. The two $j$ th transmit-antenna subsets obtained from the first step (one for each method) are $S_{\text {CRCES }}^{j}$ and $S_{\text {NRCES }}^{j}, j \in \zeta$.

In the following discussion, denote the $j$ th transmitantenna subset by $S^{j}, S^{j} \in C^{K \times L}$, independently of its origin $\left(S_{\text {CRCES }}^{j}\right.$ or $\left.S_{\text {NRCES }}^{j}\right)$. This transmit-antenna subset can be expressed as a row vector as follows:

$$
S^{j}=\left[S_{1 \bar{L}}^{j}, S_{2 \bar{L}}^{j}, \ldots, S_{K \bar{L}}^{j}\right] .
$$

To maximize the received SNR, $\gamma$ should be minimized, as per (4) and (6). Therefore, the value of $\gamma$ is calculated for each transmit-antenna subset, and the transmit-antenna subset for which $\gamma$ reaches the smallest value is selected in this second step. The value of $\gamma_{j}$ corresponding to transmitantenna subset $S^{j}$ is calculated by

$$
\gamma_{j}=\operatorname{Tr}\left\{\left(S^{j} S^{j^{H}}\right)^{-1}\right\},
$$

where $\left(S^{j} S^{j H}\right)^{-1}$ is

$$
\left(S^{j} S^{j H}\right)^{-1}=\left[\begin{array}{ccccc}
\left(S_{1 \bar{L}}^{j}\right)^{2} & \cdots & S_{1 \bar{L}}^{j} S_{k \bar{L}}^{j} & \cdots & S_{1 \bar{L}}^{j} S_{K \bar{L}}^{j} \\
\vdots & \ddots & \vdots & \ddots & \vdots \\
S_{k \bar{L}}^{j} S_{1 \bar{L}}^{j} & \cdots & \left(S_{k \bar{L}}^{j}\right)^{2} & \cdots & S_{k \bar{L}}^{j} S_{K \bar{L}}^{j} \\
\vdots & \ddots & \vdots & \ddots & \vdots \\
S_{K \bar{L}}^{j} S_{1 \bar{L}}^{j} & \cdots & S_{K \bar{L}}^{j} S_{k \bar{L}}^{j} & \cdots & \left(S_{K \bar{L}}^{j}\right)^{2}
\end{array}\right]^{-1}
$$

and $S_{k \bar{L}}^{j}$ is the $1 \times L$ row channel vector from the $L$ transmit antennas to the $k$ th user with subset $S^{j} ;\left(S_{k \bar{L}}^{j}\right)^{2}$ can be expressed as

$$
\left(S_{k \bar{L}}^{j}\right)^{2}=\left|\widetilde{h}_{k j}\right|^{2}+\left|\widetilde{h}_{k(j+1)}\right|^{2}+\cdots+\left|\widetilde{h}_{k(j+L-1)}\right|^{2} .
$$

Define $G^{j}=\left(S^{j} S^{j^{H}}\right)^{-1}, G^{j} \in C^{K \times K}$. According to (17), $\gamma_{j}$ can then be expressed as

$$
\gamma_{j}=\sum_{i=1}^{K} g_{i i}^{j}
$$

where $g_{i i}^{j}$ is the element located in the $i$ th column of the $i$ th row of $G^{j}$.

The value of $\gamma$ is thus calculated for all the candidate transmit-antenna subsets, and the transmit-antenna subset resulting in the smallest value of $\gamma$ is selected as follows:

$$
j^{*}=\underset{j \in \zeta}{\operatorname{argmin}}\left\{\gamma_{j}\right\}
$$

where $j^{*}$ is the index of the selected transmit-antenna subset. In this way, the optimal transmit-antenna subsets for the CRCES and NRCES methods, $S_{\text {CRCES }}^{j^{*}}$ and $S_{\text {NRCES }}^{j^{*}}$, are selected.

In the proposed methods, the criterion to select the candidate transmit-antenna subsets is SNR maximization. Therefore, the advantages of the proposed methods will be more noticeable at low SNR; as the SNR increases, the differences from the existing methods will decrease. Among the proposed methods, CRCES has the particularity of being focused on improving performance by considering capacity first. Therefore, it not only shows the highest performance, but also exhibits a performance difference from the conventional method that is even greater at low SNR.

\section{Performance Evaluation}

This chapter compares the performance and complexity of the proposed algorithms with those of existing methods: capacity-based reduced-complexity selection (CBRCS) [11], norm-based selection (NBS) [12], and no antenna selection $(N=L)$. The Shannon spectral efficiency (SE) for the transmit-antenna selection schemes is evaluated and compared. It is assumed that the transmit power is the same for all antennas. The signal to interference plus noise ratio (SINR) of user $k$ considering only the BS is calculated as follows:

$$
\operatorname{SINR}_{k}=\frac{P_{d}\left|H_{k \bar{N}} v_{k}\right|^{2}}{1+P_{d} \sum_{i=1, i \neq j}^{K}\left|H_{i \bar{N}} v_{i}\right|^{2}} .
$$

Therefore, the sum-rate $R_{k}$ and spectral efficiency SE of the $k$ th user are calculated as follows:

$$
\begin{aligned}
& R_{k}=\log _{2}\left(1+\operatorname{SINR}_{k}\right), \\
& \mathrm{SE}=\sum_{k=1}^{K} R_{k} .
\end{aligned}
$$

Figure 2 shows the results of the spectral efficiency analysis for massive MIMO systems with different antenna selection schemes. The number of antennas $(N)$ of the BS is 64 , the number of users $(K)$ is 10 , and the number of RF chains $(K)$ is also 10. A perfect Rayleigh fading channel is assumed. The precoding method is $\mathrm{ZF}$.

The exhaustive search requires $C_{K}^{N}$ operations [8], which implies immensely large simulation times as the number of antennas increases; therefore, its actual implementation is not possible and the method is excluded from the analysis. As shown in Figure 2, the spectral efficiencies of CRCES and NRCES were $5.2 \mathrm{bit} /(\mathrm{s} \cdot \mathrm{Hz})$ and $26.5 \mathrm{bit} /(\mathrm{s} \cdot \mathrm{Hz})(\mathrm{CRCES})$ and $3.6 \mathrm{bit} /(\mathrm{s} \cdot \mathrm{Hz})$ and $18.6 \mathrm{bit} /(\mathrm{s} \cdot \mathrm{Hz})(\mathrm{NRCES})$ for SNRs of $0 \mathrm{~dB}$ and $10 \mathrm{~dB}$, respectively. In these two SNR conditions, CRCES showed improvements of $1 \mathrm{bit} /(\mathrm{s} \cdot \mathrm{Hz})$ and $0.2 \mathrm{bit} /(\mathrm{s} \cdot \mathrm{Hz}) \mathrm{rel}-$ ative to CBRCS (resp.), and NRCES showed improvements of $1.9 \mathrm{bit} /(\mathrm{s} \cdot \mathrm{Hz})$ and $8.1 \mathrm{bit} /(\mathrm{s} \cdot \mathrm{Hz})$ relative to $\mathrm{NBS}$ (also resp.). Compared with the no antenna selection case at $\mathrm{SNR}=10 \mathrm{~dB}$, CRCES and NRCES showed good performance improvements $(17.3 \mathrm{bit} /(\mathrm{s} \cdot \mathrm{Hz})$ and $9.5 \mathrm{bit} /(\mathrm{s} \cdot \mathrm{Hz})$, resp.). 


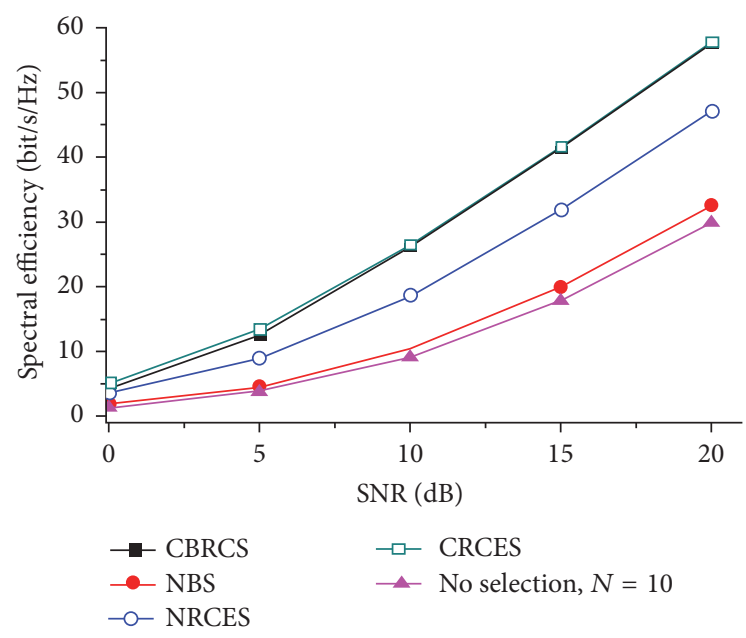

FIGURE 2: Spectral efficiency of the different antenna selection schemes $(N=64, K=10$, and $L=10)$ as a function of the SNR.

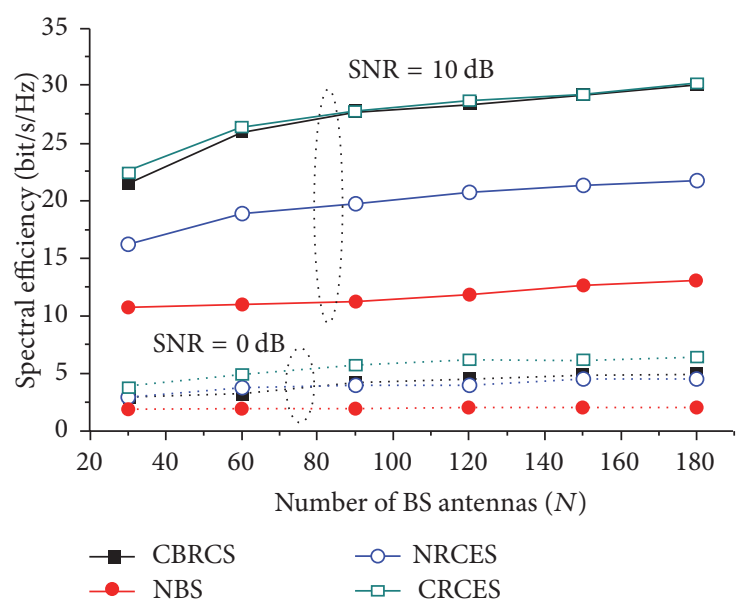

FIGURE 3: Spectral efficiency of the different antenna selection schemes for an increasing number of BS transmit antennas; $K=10$, $L=10$.

Figure 3 shows the spectral efficiency behavior as a function of the number of transmit antennas $(K=10, L=10)$. When $\mathrm{SNR}=0 \mathrm{~dB}$ and the number of antennas was 60 or less, the spectral efficiencies of the CRCES and NRCES schemes were approximately $1.7 \mathrm{bit} /(\mathrm{s} \cdot \mathrm{Hz})$ higher than those of CBRCS and NBS, respectively. The performance difference between NRCES and CBRCS was approximately $0.4 \mathrm{bit} /(\mathrm{s} \cdot \mathrm{Hz})$. When $\mathrm{SNR}=10 \mathrm{~dB}$, NRCES showed a performance approximately $8 \mathrm{bit} /(\mathrm{s} \cdot \mathrm{Hz})$ higher than NBS. CRCES, on the other hand, showed an approximate $0.4-1 \mathrm{bit} /(\mathrm{s} \cdot \mathrm{Hz})$ performance gain relative to CBRCS. Similarly, the CRCES scheme produced more effective results at lower SNR, even when the number of antennas increased.

In the case of CBRCS, $L$ optimal antennas are selected by (7) to (8); $S_{\text {CRCES }}^{1}=\left[\widetilde{H}_{\bar{K} 1}, \widetilde{H}_{\bar{K} 2}, \ldots, \widetilde{H}_{\bar{K} L}\right]$ is the optimal transmit-antenna subset of CBRCS. In an ideal environment with no noise, both CBRCS and CRCES are likely to have the same antenna subset. However, in the case of low SNR

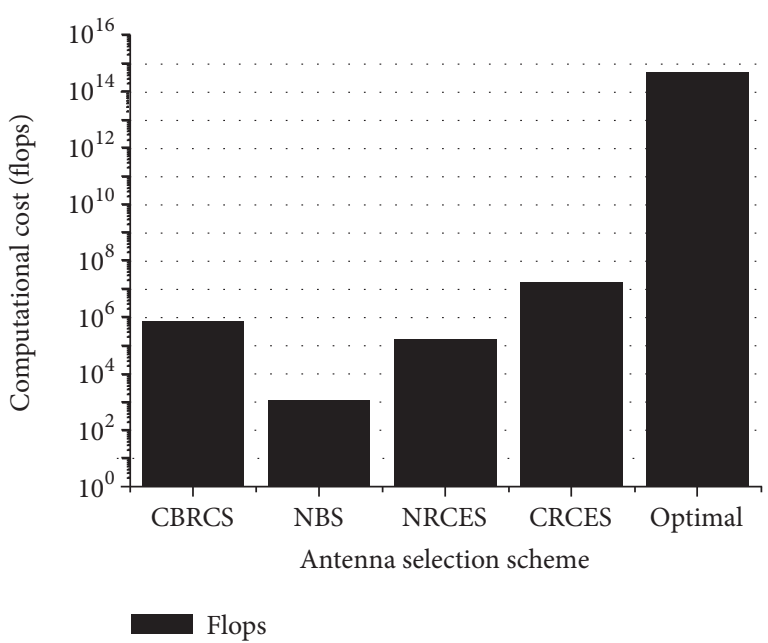

FIGURE 4: Computational complexity comparison; $N=64, K=10$, and $L=10$.

channels, the SE value for the subset selected in CBRCS does not always guarantee the largest value, because of the noise. This was expected, given that CRCES obtains the transmitantenna subsets by calculating the largest channel capacity, and then selects the optimal transmit-antenna subset (the one that maximizes the received SNR for all users). Therefore, when compared to CBRCS, the advantageous results of the CRCES scheme become more effective at low SNR. Looking at the $1.7 \mathrm{bit} /(\mathrm{s} \cdot \mathrm{Hz})$ difference from the viewpoint of total throughput, it represents $17 \mathrm{Mbit} / \mathrm{s}$, based on a mobile communication frequency band of $10 \mathrm{MHz}$. The difference becomes larger as the bandwidth increases.

Finally, to compare the different selection schemes from a computational complexity perspective, we analyzed the total search effort required by each scheme to locate the optimum transmission antennas. Table 1 and Figure 4 show the computational complexity of the transmit-antenna selection schemes in massive MIMO systems with $N=64, K=10$, and $L=10$. The computational cost of the exhaustive search is $2.9 \times 10^{14}$, which corresponds to the highest effort. The CBRCS score is $7.5 \times 10^{5}$, the NBS score is $1.2 \times 10^{3}$, and, finally, the CRCES and NRCES scores are $1.7 \times 10^{7}$ and $1.7 \times 10^{5}$, respectively.

CRCES is designed to maximize performance, whereas NRCES is designed to improve performance in terms of complexity. As a result, the NBS scheme has the lowest computational complexity, but it obtains the worst performance. Referring to Figures 3 and 4, NRCES increased the efficiency by $0.5 \mathrm{bit} /(\mathrm{s} \cdot \mathrm{Hz})$ at low SNR, despite having a computational complexity that is approximately 4.4 times lower than that of CBRCS. Therefore, when prioritizing performance, the CRCES method is excellent; on the other hand, the NRCES method is the best choice when priority is given to complexity considerations. Compared with CBRCS and NBS, the computational complexity of the proposed methods was only slightly higher, even though performance was greatly improved. 
TABLE 1: Computational complexity comparison.

Antenna

selection

scheme

$\begin{array}{lc}\begin{array}{l}\text { Exhaustive } \\ \text { search [8] } \\ \text { CBRCS [11] }\end{array} & C_{L}^{N} \cdot\left\{K L^{2}+L\left(K-\frac{L}{2}\right)-\frac{L}{2}+2 K^{2}+\frac{2}{3} K^{3}\right\} \\ \begin{array}{lr}\text { NBS [12] } \\ \text { CRCES }\end{array} & \sum_{i=N-L+1}^{N} i \cdot\left\{K(N-i+1)^{2}+(N-i+1)\left(K-\frac{(N-i+1)}{2}\right)-\frac{(N-i+1)}{2}+2 K^{2}+\frac{2}{3} K^{3}\right\} \\ \text { NRCES } & \sum_{i=2}^{N} i \cdot\left\{K(N-i+1)^{2}+(N-i+1)\left(K-\frac{(N-i+1)}{2}\right)-\frac{(N-i+1)}{2}+2 K^{2}+\frac{2}{3} K^{3}\right\} \\ & +(N-L+1) \cdot\left\{K L^{2}+L\left(K-\frac{L}{2}\right)-\frac{L}{2}+2 K^{3}+K^{2}+K-1\right\}\end{array}$

\section{Conclusion}

In this paper, we proposed two enhanced transmit-antenna selection schemes for multiuser massive MIMO systems. The performance of the proposed methods was compared with that of existing methods through spectral efficiency analysis. We also analyzed the resulting computational complexity. The simulation results show that the proposed CRCES scheme has the highest spectral efficiency and performance level, especially at low SNR. From the point of view of throughput, it provides a gain of up to $17 \mathrm{Mbit} / \mathrm{s}$ (based on the mobile communication frequency band of $10 \mathrm{MHz}$ ). The proposed NRCES scheme also shows higher performance than the existing NBS scheme. In terms of complexity, the number of searches required for the proposed schemes is slightly higher than those of the existing CBRCS and NBS methods but is significantly reduced compared to the exhaustive search option. In future work, we will expand the scope of our research into multicell scenarios.

\section{Conflicts of Interest}

The authors declare that there are no conflicts of interest regarding the publication of this paper.

\section{Acknowledgments}

This work was supported by the ICT R\&D Program of MSIP/IITP [2016-0-00183, Development of 5G Mobile Access Platform Technology Based on Virtualization Converged with Computing].

\section{References}

[1] J. F. Monserrat, D. Martín-Sacristán, J. Cabrejas-Peñuelas, D. Calabuig, S. Garrigas, and N. Cardona, "On the way towards fourth-generation mobile: 3GPP LTE and LTE-advanced," Eurasip Journal on Wireless Communications and Networking, vol. 2009, Article ID 354089, 2009.

[2] E. G. Larsson, O. Edfors, F. Tufvesson, and T. L. Marzetta, "Massive MIMO for next generation wireless systems," IEEE Communications Magazine, vol. 52, no. 2, pp. 186-195, 2014.
[3] F. Rusek, D. Persson, B. K. Lau et al., "Scaling up MIMO: opportunities and challenges with very large arrays," IEEE Signal Processing Magazine, vol. 30, no. 1, pp. 40-60, 2013.

[4] C. Shepard, H. Yu, N. Anand et al., "Argos: practical manyantenna base stations," in Proceedings of the 18th Annual International Conference on Mobile Computing and Networking, MobiCom 2012, pp. 53-64, Istanbul, Turkey, August 2012.

[5] C. Shepard, H. Yu, and L. Zhong, "Argosv2: a flexible manyantenna research platform," in Proceedings of the 19th Annual International Conference on Mobile Computing and Networking, MobiCom 2013, pp. 163-165, Miami, Fla, USA, October 2013.

[6] H. Li, L. Song, and M. Debbah, "Energy efficiency of large-scale multiple antenna systems with transmit antenna selection," IEEE Transactions on Communications, vol. 62, no. 2, pp. 638647, 2014.

[7] K. Ntontin, M. D. Renzo, A. I. Perez-Neira, and C. Verikoukis, "A low-complexity method for antenna selection in spatial modulation systems," IEEE Communications Letters, vol. 17, no. 12, pp. 2312-2315, 2013.

[8] A. F. Molisch and M. Z. Win, "MIMO systems with antenna selection," IEEE Microwave Magazine, vol. 5, no. 1, pp. 46-56, 2004.

[9] A. F. Molisch, M. Z. Win, and J. H. Winters, "Reduced-complexity transmit/receive-diversity systems," IEEE Transactions on Signal Processing, vol. 51, no. 11, pp. 2729-2738, 2003.

[10] M. Gharavi-Alkhansari and A. B. Gershman, "Fast antenna subset selection in MIMO systems," IEEE Transactions on Signal Processing, vol. 52, no. 2, pp. 339-347, 2004.

[11] P. Lin and S. Tsai, "Performance analysis and algorithm designs for transmit antenna selection in linearly precoded multiuser MIMO systems," IEEE Transactions on Vehicular Technology, vol. 61, no. 4, pp. 1698-1708, 2012.

[12] J. Zheng and J. Chen, "Further complexity reduction for antenna selection in spatial modulation systems," IEEE Communications Letters, vol. 19, no. 6, pp. 937-940, 2015.

[13] Q. H. Spencer, C. B. Peel, A. L. Swindlehurst, and M. Haardt, "An introduction to the multi-user MIMO downlink," IEEE Communications Magazine, vol. 42, no. 10, pp. 60-67, 2004. 


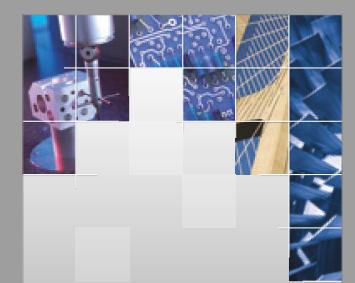

\section{Enfincering}
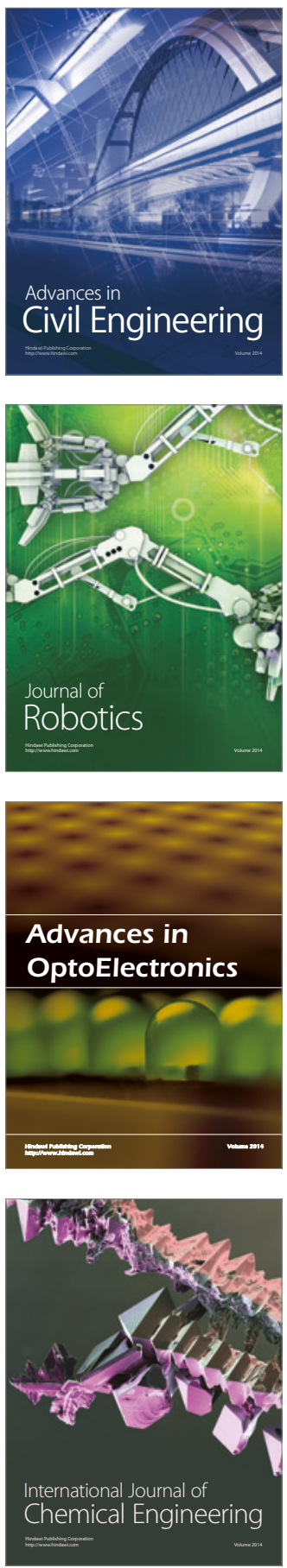

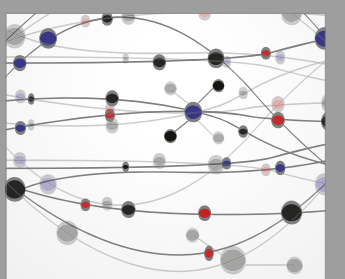

The Scientific World Journal

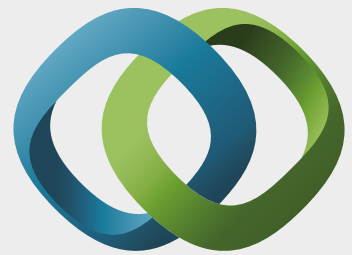

\section{Hindawi}

Submit your manuscripts at

https://www.hindawi.com
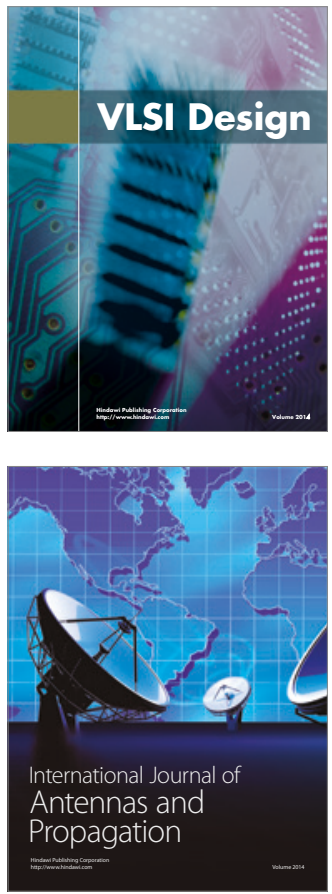

\section{Rotating}

Machinery
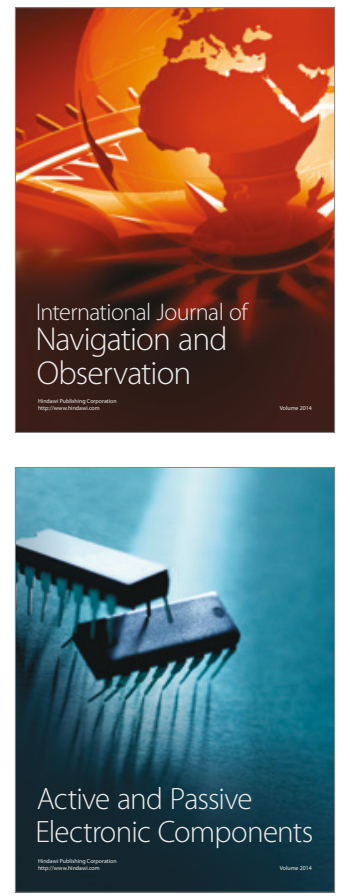
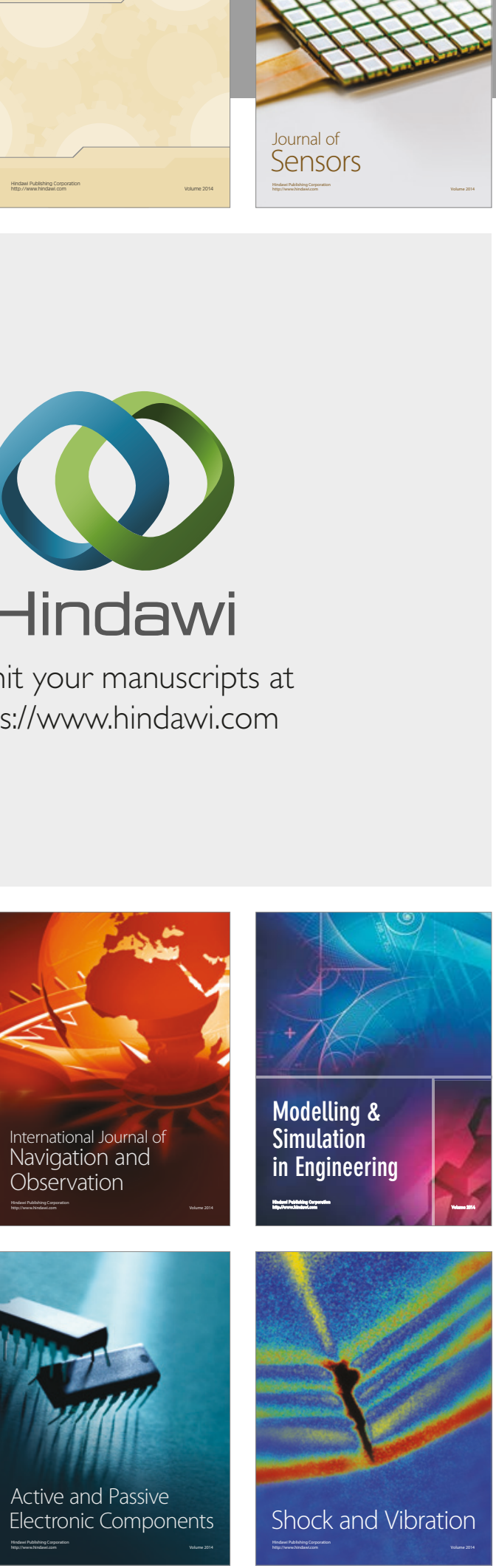
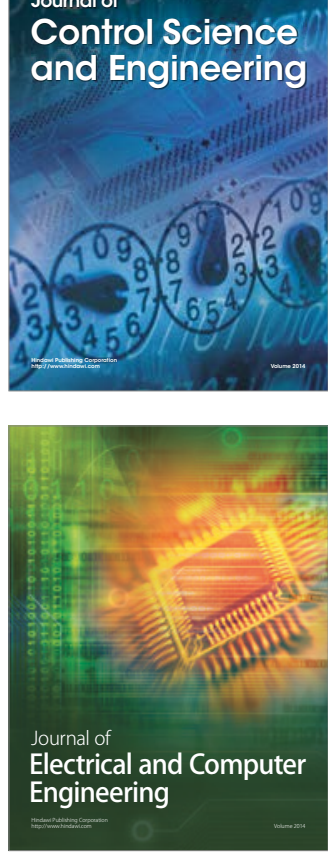

Distributed

Journal of

Control Science

and Engineering
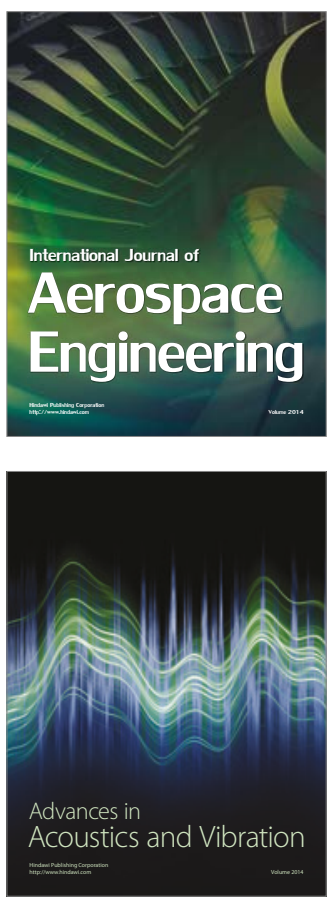

Sensor Networks 